\title{
The Application of Virtual Simulation Experiment in Computer Teaching Bo Li ${ }^{1,2, a}$ and Xiuli $\mathrm{Li}^{1,2, \mathrm{~b}}$ \\ ${ }^{1}$ School of Information Engineering, Zhengzhou University of Industrial Technology, Zhengzhou Henan 451150,PR China \\ ${ }^{2}$ Industrial Robot Applied Technology Institute, Zhengzhou University of Industrial Technology, Zhengzhou Henan 451150,PR China \\ a357696640@qq.com, ${ }^{b} 2051665100 @ q q . c o m$
}

Keywords: Virtual reality; Virtual simulation experiment; Computer theory teaching; Unity3D; teaching design

\begin{abstract}
With the development of computer graphics and simulation technology, virtual reality technology has been applied more and more widely in higher education. In this paper, the content of computer teaching theory is abstract, and the gap between the actual operation and students are not good understanding. The use of virtual simulation experiment as auxiliary teaching, make students immerse to carry on the simulation and operation in the virtual scene, the introduction of a new teaching mode for the theory of computer teaching. According to the questionnaire survey, the new teaching model is beneficial to improve the quality of teachers' teaching and enhance students' autonomous learning ability.
\end{abstract}

\section{Introduction}

Virtual Reality (VR) was proposed by J. Lanier of the United States in 1989. It is a collection of simulation technology and computer graphics, man-machine interface technology, and multimedia technology ${ }^{[1]}$. With the maturing of VR technology, the application value of Virtual Simulation Experiment (VSE) in the field of education is also highlighted. Apart from its application to scientific research in universities, it also has high utilization and easy maintenance in auxiliary experimental teaching. And many other advantages ${ }^{[2]}$. In computer theory teaching, the use of VES-assisted instruction enables learners to reach beyond the simple symbolization of knowledge, but is immersed in a computer-structured multidimensional information environment, relying on their own cognitive and cognitive abilities to acquire knowledge in all directions. And interact with each other. At present, VSE has been applied in computer teaching. The literature ${ }^{[3,4]}$ comprehensively analyzes the advantages of virtual simulation experiment technology in computer teaching, but it lacks actual development process and application cases.

This article first introduces the characteristics of VR technology and the advantages and value of VSE in computer teaching. Then it gives the design and development process of VSE. The entire process includes the selection of development preparation, experiment content and development tools, and later uses the unity3D virtual engine to achieve virtual simulation experiments. Finally, VSE was applied to the course "University Computer Foundation". Through the teaching effect feedback and questionnaire survey results, it was shown that VSE as an auxiliary teaching method can vividly and vividly draw abstract and incomprehensible knowledge points in computer theory learning. The effect of "introducing bricks and attracting jade" enhances teachers" teaching quality and teaching atmosphere, and at the same time increases learners' interest in learning and self-learning ability.

\section{The Design and Implementation of Virtual Simulation Experiment}

In The Early Stage Of The Design And Development Of The Preparatory work, according to the access to a large number of VR technology and simulation experiment, computer aided instruction (MCAI), education and psychology of literature and international journals, the application of VR technology in computer teaching and the design of VSE related development tools ${ }^{[5-7]}$. 


\section{Experiment Content Selection}

In recent years, computer technology has been developing rapidly. Internet technology and Internet are changing people's life, learning and working way at an amazing speed. The course of the computer foundation of the university is to make the contemporary college students master the computer knowledge system to meet the requirements of the development of the times. In the "University Computer Foundation" course teaching, with Computational Thinking oriented, problem solving ability as the goal, from the theory and methodology systematically expounds the features and problems of computer basic application of computer knowledge, so that students can solve practical problems in life. Because of the popularity of university education, the number of students is increasing, and the existing experimental equipment can not meet the needs of teaching. The application of VSE technology in the course of University Computer Foundation enables learners to learn the same experience with real scenes at any place, which not only enhances students' learning autonomy and enthusiasm, but also lightens the burden of school teaching.

\section{Development Tools Selection}

\section{Virtual Reality Development Engine - Unity3D}

The core tool of virtual simulation experiment development - Unity3D. Unity3D is a development tool of integrated multi platform game by a Unity game player Technologies developed easily create 3D video games, architectural visualization, real-time 3D animation and other types of interactive content and virtual reality, is a fully integrated professional game, virtual reality development engine [8].

The features of the Unity3D virtual reality engine:

The visual programming interface completes all kinds of development work, efficient script editing, and convenient development;

Automatic instant import, Unity supports most 3D models, bones and animations are imported directly, texture materials are automatically converted to U3D format;

Multi-platform development and deployment of works can be completed with just one click;

The bottom layer supports OpenGL and Direct11, simple and practical physics engine, high quality particle system, easy to get started, and the effect is fidelity;

Support Java Script, C \#, Boo scripting language;

Unity has excellent performance, outstanding development efficiency and great cost-effectiveness;

\section{D Modeling Software - 3ds Max}

The3D Max is a 3D animation rendering and production software based on PC system developed by Autodesk company, It has a very strong function and distinctive characteristics. It has involved in game animation, architectural animation, interior design, film and television animation, industrial design and so on.

First of all, 3ds Max has good expansibility. The modeling function is very powerful, and it can be compatible with a large number of plug-ins. Secondly, it is easy to operate and easy to handle. Finally, its powerful rendering function can make the effect very realistic.

\section{Image Processing Software - Photoshop}

Photoshop is Adobe's most influential software, a set of graphics and image processors for image scanning, editing, image editing, creative advertising, and image input and output. It is popular among graphic designers and computer art enthusiasts. Like it; its application involves advertising photography, graphic design, artistic text, web page production, photo design, interface design and other aspects.

\section{Design and Development Process of Virtual Simulation Experiment}

Through the research and analysis of the functions of each module, in the specific development process, the model and the texture map in the virtual scene are all derived from the real scene. The material texture map and the plane model of the real scene are acquired in advance through the camera and processed through Photoshop and 3dmax. Texture and build a 3D model of the real 
scene, then import it into Unity3D to build the virtual platform, set up the rendering on the Unity3D platform via sound effects, graphical interfaces, plugins, lighting, write interactive code, and finally publish the settings, as shown in Figure 1.

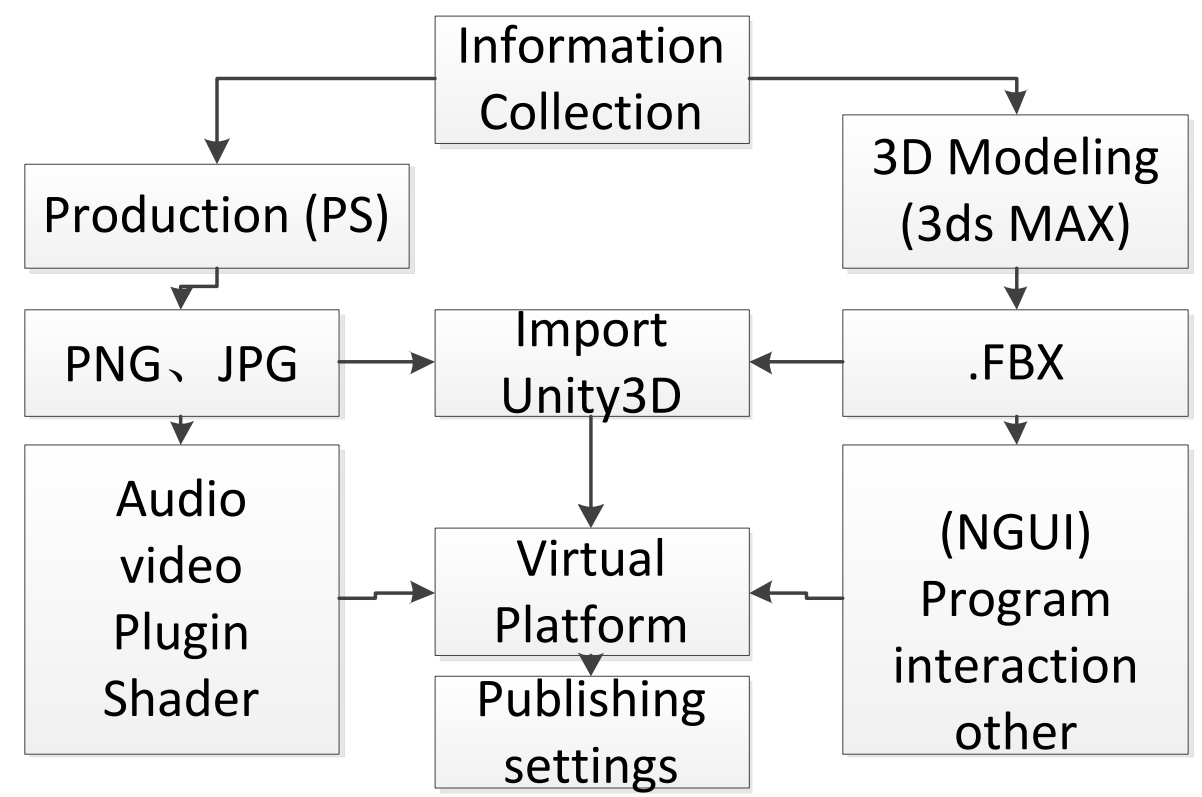

Figure 1. Development flow chart of virtual simulation experiment

\section{Application of Virtual Simulation Experiment in "University Computer Foundation" Course}

The development of virtual simulation system, so that students can prepare for class and after-school self-study consolidation in the "University Computer Foundation" course, let the teacher more fascinating, increase the fun in the classroom, so as to fully play a supporting role in the virtual simulation experiment of "University Computer Foundation" in teaching.

The Interface between Virtual Simulation Experiment and Theoretical Class

The virtual simulation experiment interacts with the theoretical teaching. The essence of the virtual simulation experiment is that the theoretical teaching of experimental teaching is compatible with the theoretical teaching. The model is "teacher presentation-student watching/using computer operation-teachers explain and elicit corresponding knowledge-Corresponding exercise", As shown in Figure 2. Compared with the point of knowledge that teachers directly put out of this section of the classroom, it allows students to have a process of positive thinking and self-derivation of experimental principles.

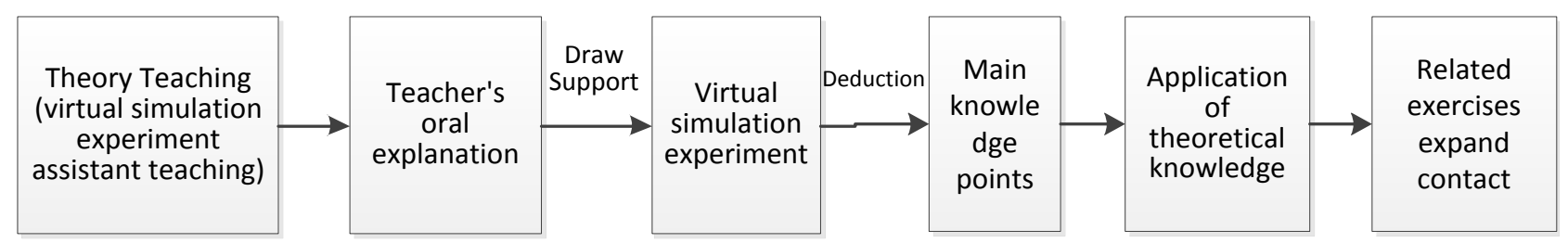

Figure 2. Flow chart for deriving knowledge points from virtual simulation experiments

\section{Teaching Design of Virtual Simulation Experiment-aided Teaching}

In the actual teaching process, teachers need to embed virtual demonstration experiments into computer teaching practice smoothly. Teachers need to do a proper teaching design before teaching practice. Instructional design involves pre-teaching design, learning resource design, and teaching. The design of situational design and learning evaluation tools, and the specific process is shown in Figure 3. 


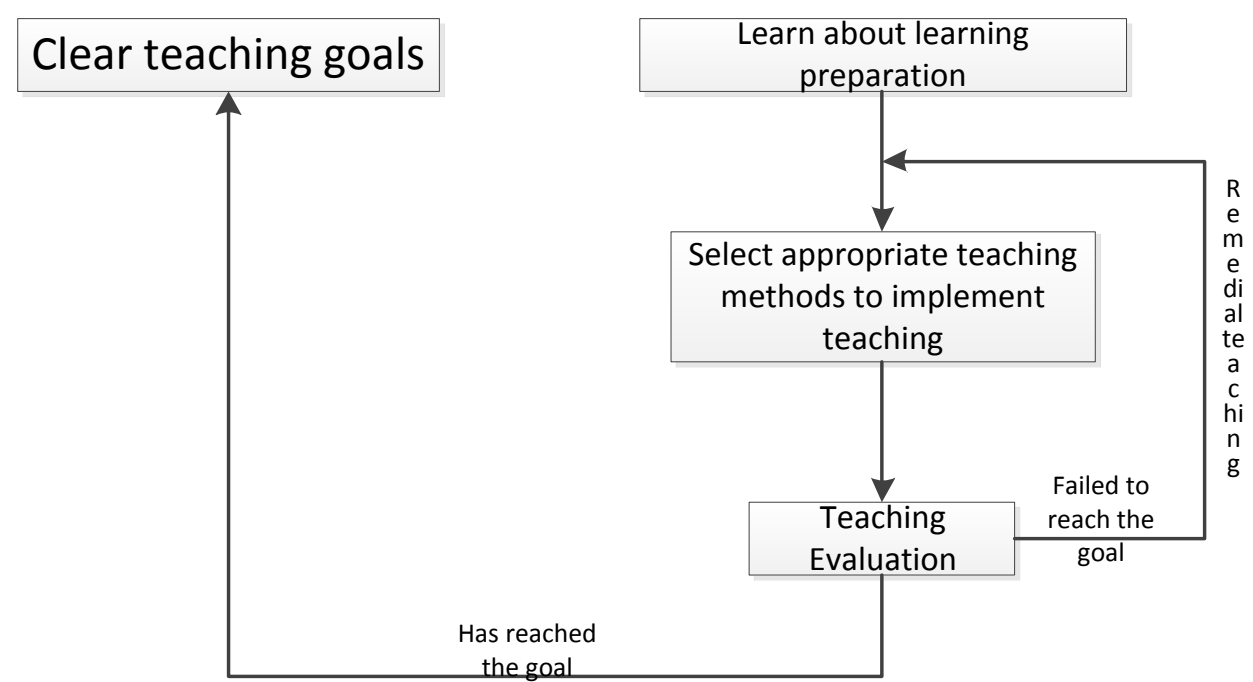

Figure 3. Flow chart of virtual simulation experiment-assisted instructional design

\section{Application of Virtual Demonstration Experiment in Computer Teaching}

By way of questionnaire in the selected industrial Institute of technology Zhengzhou two learning similar undergraduate classes as test samples, through questionnaire, and the results of the questionnaire evaluation index analysis, make the corresponding evaluation and Reflection on the application of virtual simulation in computer teaching in the classroom.

\section{Evaluation Process}

Research Purpose

Through the questionnaire to understand the application of virtual simulation experiments in computer teaching.

Research Methods

Questionnaire surveys, comparative studies, quantitative studies, etc.

Sample Selection

In the first class and the second class, the scores of the two classes are basically the same before the test. Then, for the teaching method of the embedded virtual demonstration experiment in the class division, the second class of the division includes traditional teaching methods. The total number of students in one class is 46 , and the second class is 48 .

Test Time

February 2017 to July 2017, a six-month period.

Evaluation Content

Through half a year of experimental teaching, the main contents of this evaluation involve: students' interest in the "University Computer Basics" course, their corresponding experimental operation skills, and their awareness of theoretical issues

\section{Evaluation Results}

In this questionnaire survey, a total of 94 questionnaires were issued for two classes and 89 valid questionnaires were returned. Among them, 43 questionnaires were valid for one class, 46 questionnaires were for second class, and the recovery rate was $94.7 \%$.

Through the research and statistics of the questionnaire, the results are as follows:

$50.5 \%$ of survey respondents are very optimistic about the combination of virtual simulation experiment and theoretical teaching;

According to a class of questionnaires, $58.70 \%$ of students think that they are very helpful in their own learning. This to a certain extent also shows that virtual simulation experiments have an auxiliary role in promoting computer learning. On the other hand, $69.57 \%$ of students believe that 
virtual simulation experiments can be used as a good inspiration for pre-classification and after-school consolidation. Through the use of virtual situations, the knowledge of learners' own reserves is mobilized, and students are better understood and familiarized with. Theoretical knowledge.

Evaluation of teaching practice in virtual simulation experiment system Survey results: Nearly $73.03 \%$ of respondents are very optimistic about the combination of virtual simulation and experimentation theory teaching. They believe that the combination of virtual simulation experiment and theoretical teaching increases students' theoretical knowledge. Learning interest.

\section{Concluding Remarks}

As a new technology in the forefront of science and technology, virtual reality technology has defects such as poor hardware and software performance, high cost and poor experience. It is not mature to popularize this technology in university teaching. However, it is undeniable that with the improvement of technology and combination of virtual reality technology and education, it has wide prospects. The virtual simulation experiment with the "University Computer Foundation" curriculum teaching together gives a new teaching mode, the characteristics of the virtual simulation experiment of immersion and interactivity in the classroom to play a "brick" effect of the "University Computer Foundation" course teaching in the abstract, not easy to understand the content vivid and interesting. Finally, through the questionnaire evaluation and feedback, the new teaching mode combining virtual simulation experiment with computer theory teaching enhances students' interest in learning and autonomous learning ability, and improves the teaching quality of teachers.

\section{References}

[1] Zhao Qinping. A review of virtual reality [J]. Chinese Science (series F: Information Science). 2009 (01)

[2] Li Ping, Mao Changjie, Xu Jin. Developing state-level virtual simulation experiment teaching center to improve the information level of experimental teaching in Colleges and universities. [J]. laboratory research and exploration. 2013 (11)

[3] Xu Xinxin. The application of virtual reality technology VR in computer teaching [J]. information and computer (theoretical Edition). 2016 (21)

[4] Xue Yadi. Research on computer-aided teaching based on virtual reality technology [J]. Journal of Lanzhou College of Arts and Sciences (NATURAL SCIENCE EDITION). 2017 (01)

[5] Wofford M M, Wofford J L. The affordability and efficacy of MCAI. Archives of Internal Medicine, 1995,155(15):1682

[6] Zhang Aixiang. Elementary analysis of computer teaching in Colleges and universities [J]. China Science and technology information. 2013 (09)

[7] Ning Yong, Tecsun. Hole computer teaching reform of colleges and universities and the development strategy of [J]. information technology. 2013 (24)

[8] Unity Technologies. Unity 5.X from the beginning to mastery of [M]. China Railway Press, 2016 